\title{
Anabases
}

ANABASES Traditions et réceptions de l'Antiquité

$20 \mid 2014$

Varia

\section{Musique et hexamètre français}

\section{François Cam}

\section{OpenEdition}

Journals

Édition électronique

URL : http://journals.openedition.org/anabases/5073

DOI : 10.4000/anabases.5073

ISSN : 2256-9421

\section{Éditeur}

E.R.A.S.M.E.

\section{Édition imprimée}

Date de publication : 1 novembre 2014

Pagination : 257-266

ISSN : 1774-4296

\section{Référence électronique}

François Cam, « Musique et hexamètre français », Anabases [En ligne], 20 | 2014, mis en ligne le 01 novembre 2017, consulté le 20 octobre 2019. URL : http://journals.openedition.org/anabases/5073 DOl : 10.4000/anabases.5073 


\section{Musique et hexamètre français}

FranÇOIS CAM

«Paris, le 16 septembre 2003

Cher François, pourrais-tu mettre en musique le début de l'Odyssée?

Muse, dis-moi les détours de l'homme aux ruses nombreuses, détours nombreux, lorsqu'il eut détruit la cité troyenne, * sainte, et connu les murs et le cœur de mortels innombrables, et enduré sur la mer les douleurs innombrables de l'âme, pour obtenir son retour et la vie de ses compagnons d'armes.

Mais il ne put sauver, malgré son désir immense, ses compagnons, qui périrent du fait de leur propre sottise, Les insensés! Ils mangèrent les bœufs du soleil, le splendide Hypérion! Et lui les priva du retour désirable. Fille de Zeus, commence par nous raconter leur histoire.

* variante :

Muse, dis-moi, de l'homme aux ruses nombreuses les mille vagabondages, quand il eut détruit la cité troyenne..."

Voici un message QUi ME MIT IMmédiatement aU travail (chose nouvelle pour moi). D'autant qu'au verso (photocopie d'un tract du théâtre Démodocos annonçant " Une comédie antique reconstituée / musique, chœurs, masques / Les Grenouilles / d'Aristophane ») Philippe Brunet ajoutait : "Est-ce possible très vite ? Nous partons le 2 octobre à Athènes. » Il y avait aussi quatre consignes : "Recitar cantando [1], en gardant le plus 
possible l'unité des vers [2] et, si tu peux, six mesures [3]... C'est Circé ${ }^{1}$ qui chante : aujourd'hui : Agnès, mais demain ce peut être une chanteuse moins experte [4]. »

Depuis notre première collaboration artistique (Les Amours d'Arès et Aphrodite en 1996 - Homère déjà) et pour tout dire notre rencontre, je m'étais remis activement à la poésie et à la musique dans l'espoir d'une union réussie des deux qui ne fût pas seulement un parallélisme, un caprice. La mise en musique de ces hexamètres français suivant les quatre consignes me réservait une belle surprise qui est le sujet de cet article où figurent pour illustration deux partitions : la mélodie originale de 2003 écrite pour le français (fig. 2) et son adaptation aux dix premiers vers grecs de l'Odyssée réalisée d'abord oralement au cours des années qui suivirent puis notée enfin ce dimanche de Pâques 2014 (fig. 3).

\section{[1] « Recitar cantando »..}

Cette expression technique de la musique baroque s'entend comme une invitation à se placer sous le patronage très illustre de l'Académie de poésie et de musique dont les trente-neuf chansons en onze Modes de Claude Le Jeune contiennent la première tentative de musique baroque française ${ }^{2}$. Le travail des vers mesurés à l'antique ne pouvait être toutefois conduit, s'agissant de la traduction des vers homériques de Philippe Brunet, à partir de l'orthographe inventée par Baïf (" égzakte ékriture konform'a parler ${ }^{3} »$ ) dont la correspondance phonétique ne conviendrait pas à notre français moins chantant d'aujourd'hui.

Une application des traités de prosodie du vers français moderne aurait pu nous fournir un dispositif initial mélo-rythmique mais il fallait alors choisir un système accentuel. Une note de bas de page de Milner et Regnault ${ }^{4}$ déplorant que dans son article "Alexandrin pas mort " Pierre Fortassier ${ }^{5}$ ramène à seulement deux le nombre d'accents nécessaires à une juste diction du dit vers nous dissuade de tout système. Et le traitement musical promu par Arthur Honegger dans son Antigone (1922) de la consonne d'attaque pour renverser la prosodie traditionnelle en anacrouse nous fait définitivement reconnaître combien le récitatif de la langue française est chose plastique.

Les dix vers qui chantaient sur la feuille devaient retrouver par-delà toute théorie cette unité baïfienne entre une prosodie du parlé : des syllabes plus ou moins longues et des accents ; et une prosodie du chanté : une ligne mélodique et non pas des effets mélodiques de type madrigal - expérience tentée par Le Jeune au cœur du recueil

1 Circé, spectacle de la compagnie Démodocos mis en scène par Philippe Brunet, créé en 1999.

2 C. Le Jeune, Le Printemps, veuve Ballard et fils, Paris, 1603.

3 J.-A. DE BAIF, Étrénes de poézie fransoèze an vèrs mezurés, Paris, 1574.

4 Note 1, p. 147, in J.-C. Milner \& F. Regnault, Dire le vers, Seuil, Paris, 1987.

5 P. FORTASSIER, "Alexandrin le grand : Alexandrin pas mort ", in Théâtre en Europe 1, Paris, 1984, p. 76. 
Le Printemps avec la sextine Du Trist'hyver dont le style autorise à faire de Baïf son auteur $^{6}$. L'essentiel est dans le principe de cette unité et le résultat tel qu'il se donne à chanter dans la figure 1. Cette mélodie a été écrite d'un trait (seul le dernier vers témoigne d'une modification, un la à la place d'un si sur Zeus, datant de 2007, corrigeant un effet de pathos imitatif) - dans un système diatonique basique d'un ambitus de neuvième, passant outre toute chirurgie de la quantité et de l'accent des mots, dont je devenais parallèlement un strict praticien ayant engagé sur les strophes lyriques en grec ancien un travail bientôt académique. D'autres expériences sur des vers français

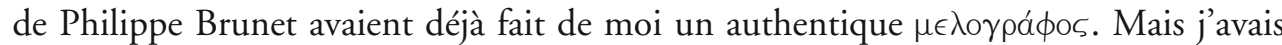
conçu alors une mélodie sur un rythme donné que j’avais ensuite adaptée, c'est-à-dire trois étapes contre une seule pour les vers de l'invocation à la Muse. La mélodie a donc débuté par un mi puis, ascendante, par la répétition de la formule $f a$, $l a$, si sur un rythme dactylique inspiré des premiers mots du texte...

«Mu-se, dis-moi les dé-tours"

... puis elle s'est déroulée, enroulée, ravivée, ralentie jusqu'à la fin, dans le temps qu'il faut pour chanter et pour transcrire, en tenant compte :

- des accents du français pour les valeurs longues, accents portant sur la dernière syllabe des groupes syntaxiques : "dis-moi, les détours " (brève-longue, brève, brève-longue) ainsi que sur certaines syllabes relevant parfois d'une fonction phatique du phrasé poétique : «Fil-le de Zeus » (quatre longues);

- et des deux principes qui ont joué à part égale avec la façon recitar cantando et dont j'explique en [2] et [3] la manière de les traiter équitablement : l'unité spécifique du vers hexamètre à marquer aussi légèrement que sûrement, et une course générale des vers qui ne fût pas oublieuse des six mesures.

$$
\text { [2]... « en gardant le plus possible l'unité des vers »... }
$$

Une familiarité théorique et pratique de l'hexamètre grec de longue date n'est pas étrangère au caractère récursif de cette mélodie très proche de la ritournelle. L'unité des vers hexamètres français traduits d'Homère pouvait être assurée aux deux bouts par : - un retour à chaque début de vers à la note initiale de base (octaviée au besoin) que nous n'appellerons pas ici la tonique mais selon sa hauteur l'hypate ou la nète des disjointes $^{7}$ : la note $m i$, en position 1 , c'est-à-dire longue au premier temps fort du vers ;

6 Cf. M. Augé-Chiquet, La vie, les idées et l'auvre de Jean-Antoine de Baïf, Toulouse, 1909.

$7 \quad$ L'échelle de cette mélodie se situe dans un trope qui est le dorien diatonique des manuels de musique antique (dans la transcription de Riemann) n'exploitant du système complet à deux octaves que le tétracorde central : $m i$-la ; le tétracorde des disjointes : si-mi; et le fa supérieur. Dans ce système les Grecs nomment hypate le mi de la première ligne et nète des disjointes le mi à l'octave supérieure. La note centrale, ou mèse, est le la, et le si la paramèse. 
- une clausule régulière dactyle + spondée, typique de l'hexamètre dactylique : sensibilisé à la scansion d'Homère en grec depuis les années de lycée, puis à celle de l'hexamètre dactylique français depuis sept ans, cette clausule n'était pas contraignante, tombait naturellement, sans que j'eusse besoin de surveiller ma mélodie ni comme cet apprenti que nous fûmes tous, lecteurs d'hexamètres, de revenir en arrière.

Outre cette unité des vers, chaque vers a son caractère ( $\dot{v \theta \mu o ́ s) ~-~ d a c t y l i q u e ~}$ ou spondaïque, et sa progression (ả $\gamma \omega \gamma \eta \dot{)})$, la mélodie peut être pour mieux en juger comprise en deux parties correspondant aux deux moitiés du passage :

- vers 1 à 5 : ascendante sur les deux premiers vers la mélodie redescend sur la première moitié du vers 3 puis remonte sur la fin. Les vers 4 et 5 sur une mélodie identique combinent les mouvements ascendants et descendants.

- vers 6 à 10 : l'adverbe d'opposition " Mais " suivi de la négation "ne put sauver» nous invite à moduler et faire cesser le rythme dactylique régulier qui animait toute

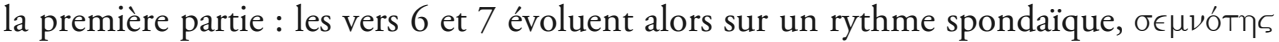
toute divine annonçant la prière finale. Les vers 8 et 9, réanimés par leurs points d'exclamation offrant une même césure initiale après quatre syllabes d'une prosodie identique ( Les in-sen-sés! » / «Hy-pé-ri-on!»), reprennent le cours dactylique et sa

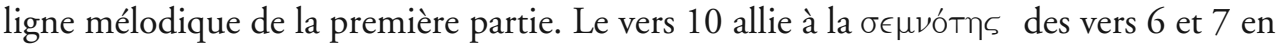
sa première partie le rythme dactylique qui emporte la fin de ce chant.

Dans la terminologie antique notamment du De Musica d'Aristide Quintilien ${ }^{8}$, l'à $\gamma \omega \gamma \eta$ de ces vers dans leur ensemble (donc de la mélodie entière) n'étant pas continue par degrés conjoints serait qualifiée de $\pi \lambda$ окń : par sauts d'intervalles. À l'exception toutefois de deux passages se répondant en miroir du vers 7 au vers $10:$ un mouvement

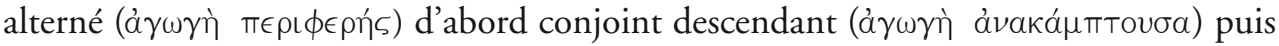

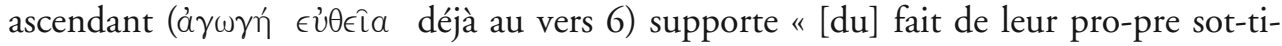
[se] " sur les quatre notes du tétracorde central au vers 7 . Lui répond au vers 10 un mouvement conjoint ascendant pour " com-men-ce par nous " qui se termine sur la note disjointe $s i$, paramèse du système, sur laquelle se termine la mélodie.

Enfin, les vers 6, 7 et 10 qui se distinguent nettement du rythme dactylique d'ensemble ont été composés dans une échelle restreinte spécifiant leur caractère : ainsi la mélodie tient pour ces trois vers dans un ambitus de sixte (mi-do) qui se ressert encore au vers 10 dans un ambitus de quinte $(m i-s i)$. Et pour qualifier harmoniquement cette différence, la note sol (lichanos du tétracorde central mi, fa, sol, la) absente des autres vers sert la progression conjointe ascendante, descendante, alternée.

La note fa qui vient juste après le $m i$ supérieur reçoit le nom de trite du tétracorde des hyperbolées.

8 Aristide Quintilien, La Musique, trad. et commentaire par F. Duysinx, Genève, Droz, 1999. 


\section{$[3] \ldots$ « si tu peux, six mesures »...}

De ces quatre consignes la plus problématique, suivie avec toute la libéralité qu'elle accordait. Car il n'était pas sans dommage d'écrire cette mélodie en arrêtant d'abord pour chaque vers les six longues aux temps forts que suggérait une dictio epica - dans la lettre de Philippe Brunet le mot hexamètre n'apparaît d'ailleurs pas. Ainsi arrive-t-il au premier vers un métrique accident : la perte d'un temps à la troisième mesure où deux longues pour " de l'homme " en place des deux brèves aurait sauvé ce premier hexamètre français de l'Odyssée. Qu'une note tenue longue sur la préposition "de » ne fût pas ici mélodiquement jouable dans le groupe phonologique « les détours de l'homme ", elle l'aurait bien été avec la variante et son antéposition du complément "de l'homme » avant le groupe nominal " les mille vagabondages ». Mais rentrer dans les possibles de cette variante pour cette raison accentuelle d'insistance permettant l'allongement de la durée c'était privilégier le schéma, et partant reconstruire à partir des positions longues des dix vers la ligne mélodique.

Or, on l'a dit en introduction, le sens de ce travail de composition, l'union d'une prosodie du dit et du chant, était de prendre ces vers et d'en extraire une musique : la contrainte qui se serait aussitôt manifestée de placer (terme grossier mais sans équivoque) les longues au risque de perdre les sentiments, qui sortent des phonèmes : de "Fil-le " au vers 10 la majesté, ainsi que de " il » au vers 6 la grandeur, de " dé-si-rable " par son suffixe au vers 9 la puissance du désir plutôt que l'impatience du besoin, et d' ' in-nombra-bles " l'impossible énumération de tant de choses qui se pressent et s'accumulent, etc.

Ce qui importait, c'était cet art extrait des mots au fil des vers, et toute sclérose du tactus casserait la mélodie là où dans la diction la scansion du vers créerait de la musique.

Par le savant équilibre recherché dans un travail de composition nous gardant de rentrer trop avant dans l'art de l'hexamètre, que Dain nommerait ses " facilités techniques 9 " dans le chapitre sur "La facture de l'hexamètre ", on chante donc ici cinq hexamètres bancals sur dix quoique cela ne rompe pas le cours de l'ensemble : deux vers à cinq mesures et demie (v. 1,2), deux vers à six mesures et demie $(v .3,10)$ et même un vers à sept mesures et demie (v. 7).

Les cinq autres comportent bien six mesures sans l'avoir cherché, et nous constatons par ailleurs qu'exactement la même ligne mélodique a servi les vers 1 et 2 et les vers 8 et 9 par l'ajout (facilité ?) de deux brèves au deuxième temps de la quatrième mesure.

$[4]$... « demain ce peut être une chanteuse moins experte "

En cette rentrée 2003 nous n'étions plus très loin de la performance collective de la lecture de l'Iliade ${ }^{10}$ en trois jours dans sa traduction hexamétrique qui devrait encore

9 A. Dain, Traité de métrique grecque, Paris, Klincksieck, 1965, p. 54-55.

10 Homère, Iliade, trad. Ph. Brunet, Paris, Seuil, 2010. 
attendre les 5, 6 et 7 mars 2005 en Sorbonne. Il devenait nécessaire pour expérimenter le passage du parlé au chanté de tester sur des petits ensembles de vers certaines échelles issues des tropes de la musique grecque antique, pour ceux des aèdes qui s'exerceraient à chanter en s'accompagnant éventuellement d'une lyre (en anticipant de 6 ans sur leur découverte en Éthiopie !). La composition de l'invocation à la Muse s'inscrit dans l'audelà d'une performance homérique française intégralement chantée à partir de mélodies de ce type se situant (à l'exception du fa supérieur) dans ce que l'on appelle l'« octave chorale " (chantable par toutes les tessitures) du mi 2 (en clé de fa) au mi 3 (en clé de sol) :

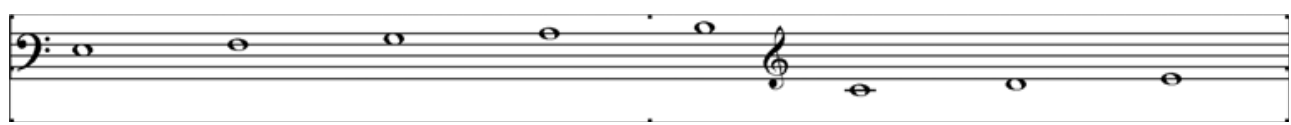

Fig. 1

Dans la transcription de Riemann, notée un demi-ton au-dessous de celle de Bellermann, cette " octave chorale " correspond à la notation du dorien diatonique, présentant l'avantage d'une ligne sans altérations à la clé. À partir du plus simple des systèmes on imagine alors sans augmentation superflue du nombre de notes des échelles diatoniques ou enharmoniques (et pourquoi pas chromatiques) dans des tropes variés ouverts aux métaboles en tous genres...

Pour la comédienne Agnès du spectacle Circé (élément des " Métamorphoses de Dionysos " du Théâtre Démodocos comprenant aussi un Orphée et une Ariane) la mélodie a été transposée à l'octave supérieure. Rien d'acrobatique mélodiquement ni modalement dans cette musique qui pût faire renoncer l'exécutante, à laquelle ne reviendrait que de faire bien entendre le poème porté par notre chansonnette.

\section{De l'hexamètre français à l'hexamètre grec : une synthèse des arts}

Ce n'est que bien plus tard que nous tentions - encouragé par notre muse, le traducteur - de chanter les hexamètres grecs avec la mélodie écrite pour le français. Et ce fut le deuxième miracle de cette expérience : de même que la mélodie française était née du texte, de même le grec renaissait dans la mélodie, dès la première tentative. Le chant rentrait dans la mesure, les mesures s'ajustaient au chant, avec aisance, en

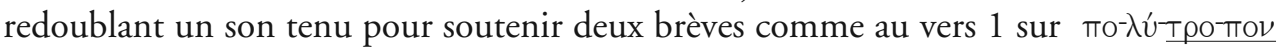
ou au vers 6 sur $\dot{\epsilon}^{-} \rho \rho v^{-} \sigma \alpha-T O$; en liant deux croches sur une syllabe longue aux vers 2 :

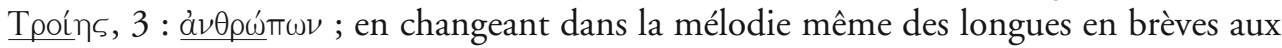
vers 7 (à trois reprises) et 10 (à deux reprises); en supprimant une note qui ne soutenait plus rien (par deux fois, aux vers 3 et 7) ${ }^{11}$. Les vers 8 et 9 conservaient intégralement

11 Pour visualiser l'ensemble des modifications, cf. fig. 3. 
la mélodie, res jucunda auditu, et apparaissent comme tels sans modifications en fig. 2 . Seule divergence forte : le vers 7 perdait son rythme spondaïque. Mais apparenté en son début au vers 6 par le groupe spondée + dactyle en miroir avec la clausule dactyle + spondée, cela leur assurait une complicité toujours sensible dans la progression de l'ensemble.

Qu'en est-il, une fois la mélodie reportée sur les vers grecs au prix des quelques ajustements soulignés, du respect de cette loi de l'accent grec commentée par les philosophes, orateurs et grammairiens de l'Antiquité jusqu'à nos jours ${ }^{12}$, qui voudrait qu'à la syllabe accentuée de chaque mot plurisyllabique corresponde dans le chant une élévation mélodique ou du moins une note dont la hauteur ne fût pas inférieure à celles portant les syllabes non accentuées du mot ? En confrontant la mélodie écrite pour le français puis appliquée au grec à la ligne accentuelle du grec pour les 52 mots plurisyllabiques accentués de ces dix vers il apparait que $60 \%$ des accents $(31$ mots exactement en acceptant au vers 6 í́ $\mu \in \nu o ́ s$ au nom d'une progression mélodique - $m i$, $f a$, sol, la - favorisant l'accent d'enclise) sont bien traités au regard de cette loi, soit une proportion très proche de celle observée généralement dans le rapport de la strophe à l'antistrophe dans les chœurs tragiques. Sans rentrer ici dans le détail de chaque vers, nous dirons donc que la ligne accentuelle des dix vers français hexamétriques de l'invocation à la Muse qui a offert cette mélodie était en correspondance avec la ligne accentuelle grecque, et que cette correspondance est un effet de l'art.

La surprise que me réservaient ces vers grecs voguant sur un air français était la force de ces correspondances : des vers français vers la musique, de la musique vers les vers grecs. Des associations de phonèmes français à celles du grec s'établissait une correspondance non verbale issue d'une rencontre que l'art seul permet. "Le traducteur-philologue fixe à l'avance, croit-il, la place de l'aède, ignorant qu'en fait c'est l'aède qui le mène ${ }^{13}$ " : le mystère révélé dans la préface de la traduction de l'Iliade me saisissait là où je l'attendais le moins. Toute la science philologico-musicale déployée pour mettre en musique ces dix vers grecs ne m'auraient pas offert la joie ressentie à cet instant. Umberto Eco dans le quatrième essai de L'CEuvre ouverte, "L'informel comme œuvre ouverte ", emprunte au domaine musical le célèbre menuet du Notenbüchlein für Anna Magdalena Bach pour illustrer « cet aspect pluriel de la communication artistique $^{14}$ ». Décrivant les premières mesures il commente : "La loi de probabilité est celle de la grammaire tonale, à laquelle la sensibilité de l'auditeur occidental post-médiéval est habitué : les intervalles y constituent plus que des différences de fréquence, ils impliquent la mise en œuvre de rapports organiques ${ }^{15}$. " Dans le cadre de notre expé-

12 Cf. A. BÉlIs, "Relation entre texte et mélodie ", in Corpus des inscriptions de Delphes, tome III : Les hymnes à Apollon, Paris, De Boccard, 1992, p. 31-32.

13 Ph. Brunet, Homère, Iliade, Paris, Seuil, 2010, p. 22.

14 U. Eco, L'Euvre ouverte, Paris, Seuil, 1979, p. 129.

15 U. ECO, L'Euvre ouverte, p. 129-130. 
rience c'est la grammaire de l'hexamètre qui a mis en œuvre les rapports organiques de la poésie et du chant, de l'aède au texte grec puis à sa traduction française puis à la mélodie et enfin au retour à Homère.

La mélodie doit-elle correspondre aux sentiments du texte, y a-t-il vraiment des longues et des brèves en français, et hors les notations autorisées, n'y a-t-il que des faussaires en musique grecque antique ? À tous ces abîmes qui s'ouvraient sous nos pas en acceptant le défi lancé par Philippe Brunet en cette rentrée 2003, l'expérience d'une telle synthèse des arts a opposé une leçon qui déterminera aussi par la suite notre approche musicale de tout système antistrophique dans la composition des odes pindariques comme des strophes lyriques des tragédies : les rapports entre musique et texte deviennent parallèles et systématiques une fois les sentiments perdus quand le rationnel efface l'irrationnel. Mais ces sentiments peuvent renaître si la prosodie et la mélodie se communiquent entre elles sous la bienveillance de l'aède les informations nécessaires à leur harmonie.

\begin{tabular}{l} 
François CAM \\
\hline ERIAC (Université de Rouen) \\
Faculté des Lettres et Sciences humaines \\
76821 Mont-Saint-Aignan cedex \\
cam.frcois@gmail.com
\end{tabular}

\section{Bibliographie}

M. Augé-Chiquet, La vie, les idées et l'auvre de Jean-Antoine de Baïf, Toulouse 1909. J.-A. DE BAIIF, Étrénes de poézie fransoèze an vèrs mezurés, Paris, 1574.

A. BÉLIS : "Relation entre texte et mélodie ", in Corpus des inscriptions de Delphes, tome III : Les hymnes à Apollon, Paris, De Boccard, 1992.

Ph. Brunet (trad.), Homère, L'Iliade, Paris, Seuil, 2010.

A. Dain, Traité de métrique grecque, Paris, Klincksieck, 1965.

F. DuYsinX, Aristide Quintilien, La Musique, trad. et commentaire, Genève, Droz, 1999.

U. Eco, L'euvre ouverte, Seuil, Paris 1979.

P. FORTASSIER, "Alexandrin le grand : Alexandrin pas mort ", in Théatre en Europe 1, Paris, 1984.

C. Le Jeune, Le Printemps, Paris, veuve Ballard et fils, 1603.

J.-C. Milner \& F. Régnault, Dire le vers, Paris, Seuil, 1987. 


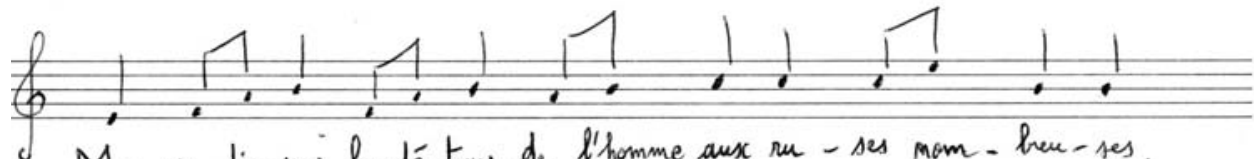

Mu-se, dis-mai les dé-touns de l'homme aux nu - ses mam - heu-ses,

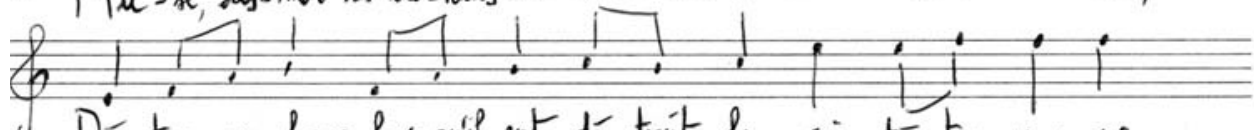

Dé-taus nom-heax lass-quil ent dé-trait la cie té tro-yen-ne,

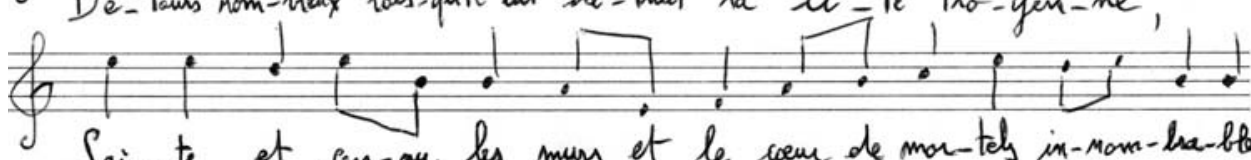
Sain-te, et can-mue les murs et le coem de mor-tels in-mam-ba-bles

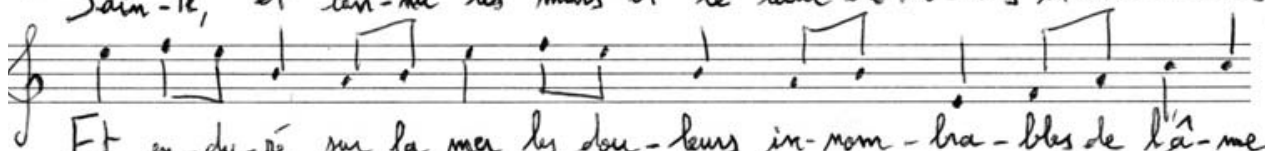
Et en-du-sé sur la ma les dou-leurs in-man-ha-bles de l'â-me,

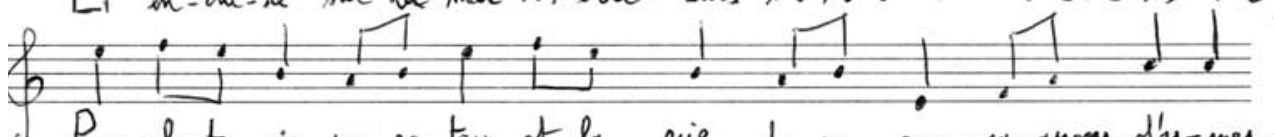
Pour ob-te-mir son re-tan of la vie de ses com-qa-gnons d'ar-mes.

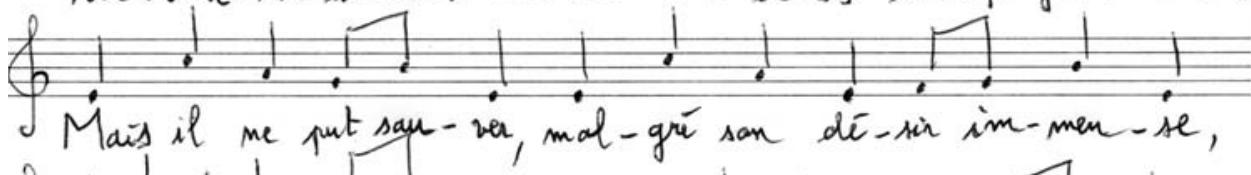

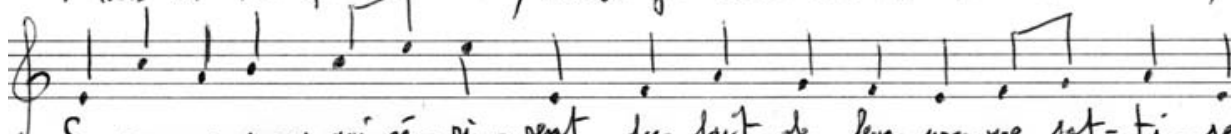

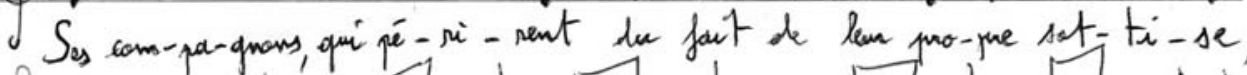

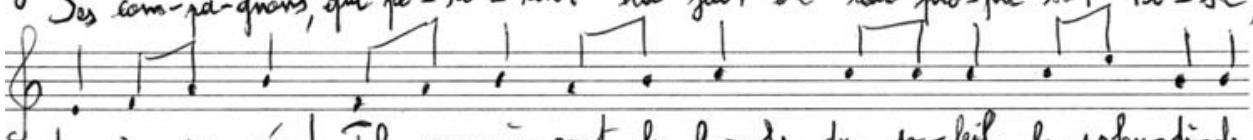
Les in-sen-sés! Ils man-gè-rent les breeufs du so-beil, le splen-di-de

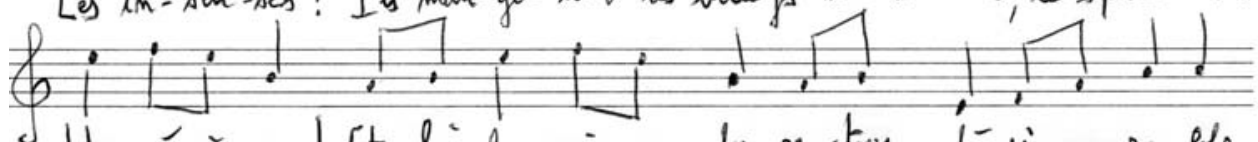

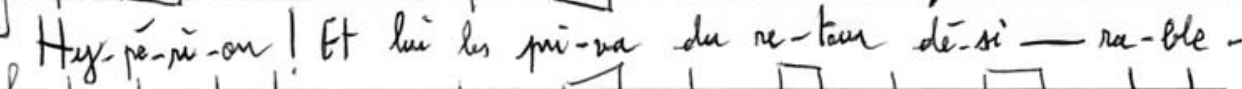

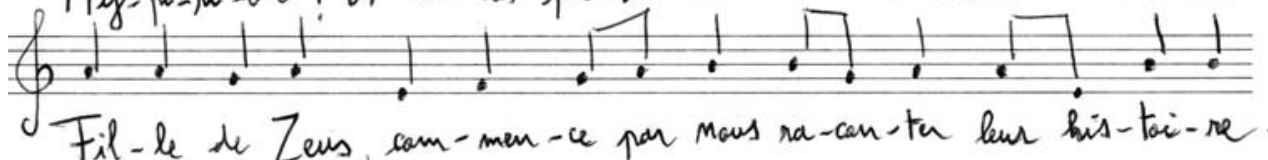

Fig. 2

Odyssée, chant I, vers 1 à 10, en français, mis en musique Traduction : Philippe Brunet Musique : François Cam 


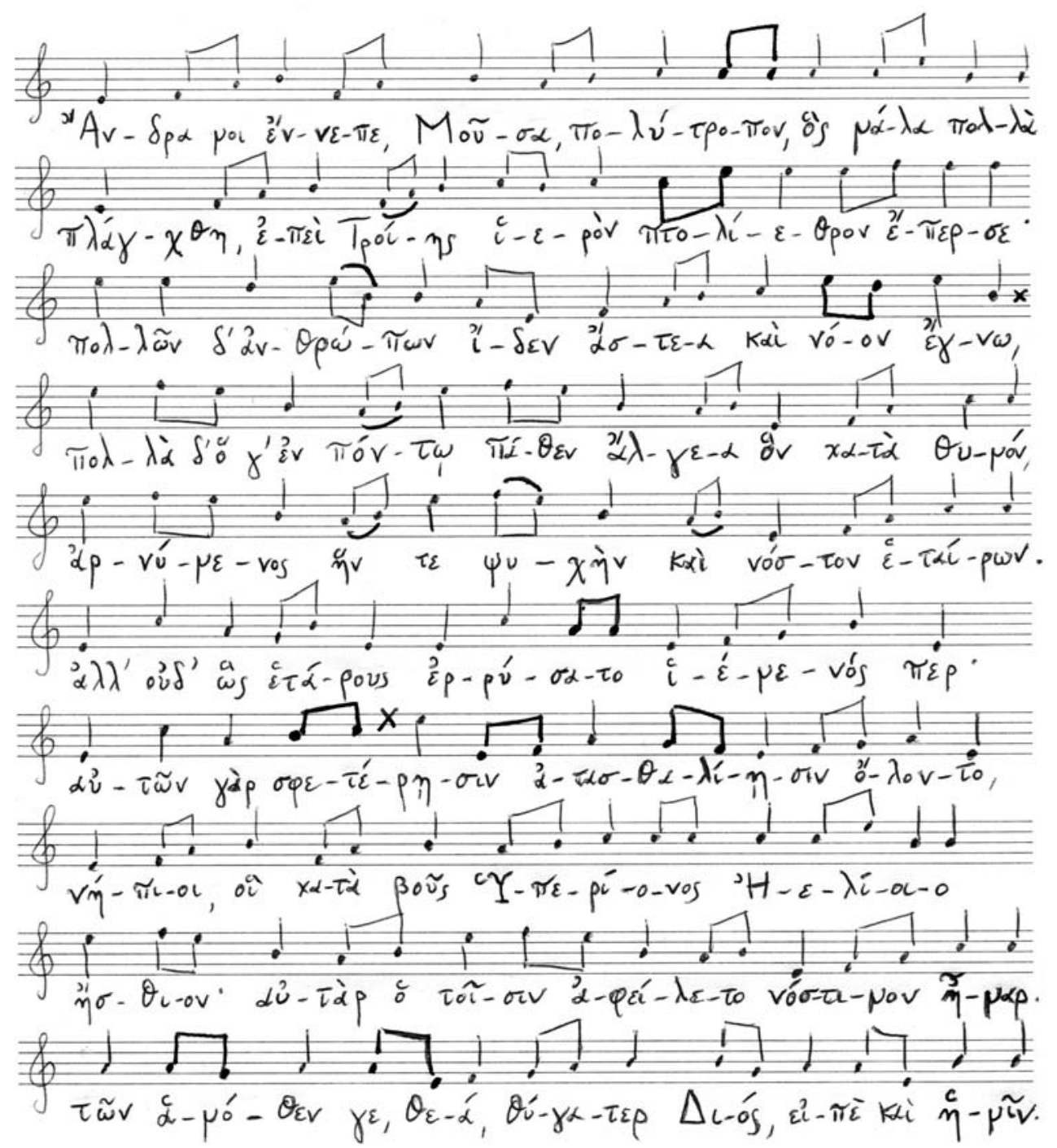

Fig. 3

Odyssée, chant I, vers 1 à 10, en grec, adaptation musicale à partir de la version française Les modifications nécessaires sont portées en gras. Elles sont de trois types:

1) deux croches pour deux syllabes brèves d'un dactyle, vers $1,2,3,6,7 \& 10$

2) une liaison pour un mouvement mélodique sur une syllabe longue, vers $2,3,4 \& 5$

3) une croix pour une note supprimée de la ligne mélodique, vers $3 \& 7$. 